\title{
$\begin{array}{ll}\text { Research Square } & \text { Preprints are preliminary reports that have not undergone peer review. } \\ \text { They should not be considered conclusive, used to inform clinical practice, }\end{array}$ or referenced by the media as validated information. \\ Pulmonary Brucellosis in Xinjiang, China: A Retrospective Study of 38 Cases and Review of the Literature
}

\section{Siqin Lan}

First Affiliated Hospital of Xinjiang Medical University

\section{Dawei Chen}

First Affiliated Hospital of Xinjiang Medical University

\section{Yuanlin He}

First Affiliated Hospital of Xinjiang Medical University

Wenya Liu

First Affiliated Hospital of Xinjiang Medical University

Hui Guo ( $\nabla$ guohui9804@126.com )

First Affiliated Hospital of Xinjiang Medical University

\section{Research Article}

Keywords: Brucellosis, Xinjiang, Pulmonary, Computed tomography

Posted Date: February 4th, 2022

DOI: https://doi.org/10.21203/rs.3.rs-1233866/v1

License: (c) (1) This work is licensed under a Creative Commons Attribution 4.0 International License. Read Full License 


\section{Abstract}

Background: Pulmonary brucellosis (PB) is a unique infection caused by Brucella and it is challenging to diagnose PB accurately for its diverse clinical manifestations. We tried to summarize PB's features to enhance our awareness of it and offer differential diagnoses in endemic zones by evaluating their demographic characteristics, clinic data, and imaging findings.

Methods: The demographic characteristics, clinic data and the imaging findings of 38 patients with PB from January 2011 to January 2020 in Xinjiang Medical University Affiliated First Hospital were presented.

Results: The median age were 51 years. The most common clinical manifestations were fever $(73.7 \%)$. The laboratory data decreased significantly, containing hematocrit (84.2\%), hemoglobin (78.9\%), red blood cell count (65.8\%), eosinophilic count (47.3\%). On computed tomography (CT), 27 patients had nodules, 23 (60.5\%) interstitial infiltration, $16(42.1 \%)$ consolidation, 15 (39.5\%) patchy shadow, and 7 $(18.4 \%)$ atelectasis. 11 patients had pleural thickening and $10(26.3 \%)$ patients suffered from hydrothorax. 20 (52.6\%) patients had lymphadenectasis in the hilum of lung or mediastinum, three pericardial effusion, and 1 pericardial thickening.

Conclusion: PB should not be excluded when a patient has chest radiologic abnormalities, meanwhile complain of an atypical high fever and fatigue, especially in the epidemic region.

\section{Key Points}

Pulmonary brucellosis is a special infection caused by brucella and it is difficult to accurately diagnose pulmonary brucellosis for its diverse clinical manifestations.

Pulmonary brucellosis seems so special that many people might ignore it, even high brucellosis incidence areas.

Pulmonary brucellosis should not be excluded when a patient has chest radiologic abnormalities, meanwhile complain of an atypical high fever and fatigue, especially in the epidemic region.

\section{Introduction}

Brucella is a small, gram-negative, intracellular aerobic bacterium that can invade a variety of organisms, including humans, wildlife (deers and zebras) and livestock (cattle and sheep) [1, 2]. Human brucellosis is an ancient zoonosis caused by Brucella, which rages worldwide, including China [2-4]. The totality of creatures it attacks is enormous, causing a magnitude of damage to both the patients and the public purse [4-6]. More than 170 countries and regions of the world have reported brucellosis. Approximately half a million people and millions of animals are diagnosed with brucellosis every year, leading to significant economic losses in public health and stock breeding $[5,7]$. 
An accident or regular encounter with the unsterilized blood, secretions, carcasses, contaminated meat, milk of infected animals may make it possible to be infected; Even inhalation of contaminated aerosols may also lead to infection [9-11]. Brucella can invade multiple organ systems [4, 5, 7, 12], especially the liver, spleen and osteoarticular system [12,13], and the lungs are rarely involved even in epidemic regions. Therefore, analyzing and summarizing PB's characteristics seemed critical, which helped to improve the cognition of PB and diagnostic accuracy.

PB seems so special that many people may overlook it, especially in low brucellosis incidence areas. It is supposed to note that Xinjiang is one of the craziest endemic regions in China due to its booming livestock industry. Therefore, we need to pay attention to the diagnosis and treatment of brucellosis. Nevertheless, studies reported by Zheng R et al. [5] showed that the distinctive and detailed clinical characteristics of PB from Xinjiang were still lacking. Our study aims to arouse PB's awareness by generalizing and summarizing demographic information, clinic data, laboratory data, CT findings, and treatment.

\section{Material And Methods}

\section{Study population}

All the case files were retrospectively reviewed to screen out the patients diagnosed with brucellosis from January 2011 to January 2020 at Xinjiang Medical University Affiliated First Hospital. PB patients' data were extracted systematically, including demographic characteristics, clinical data, imaging findings, and complications. This study was performed in accordance with the principles of the declaration of Helsinki. All participants provided written informed consent. The Ethics Review Committee ethically approved the study of the First Affiliated Hospital of Xinjiang Medical University.

Inclusion criteria were as follows: (a) They were diagnosed with brucellosis definitely with convincing bacteriological proof or a supporting result of the Standard Tube Agglutination (STA) test ( $\geqq 1: 160)$ or the positive outcome of the rose bengal plate test. (b) The imaging findings established that they had radiological abnormalities. (c) All of the clinical files and the imaging findings were complete, available and reliable.

The patients would be eliminated if they met one of the following criteria: (a) The patients suffering from lung cancer or other malignancies. (b) The patients whose clinical data or radiographic data was fragmentary. (c) The patients with respiratory dysplasia. (d) The patients exposed to other pulmonary infections like croupous pneumonia or virus pneumonia would interfere with the study. (e) The patients who were unwilling to cooperate.

\section{Laboratory protocol}

The blood samples were taken on an empty stomach in the morning before treatment. The automatic blood analyzer (Sysmex XN2000) was used for complete blood counting. We chose the Westergren 
method to test their erythrocyte sedimentation rate (ESR) and the immune turbidimetric way to test their C-reactive protein (CRP). The gold standard for brucellosis was cultivating and isolating the brucella from the pleural effusions, blood, or expectoration. The Bengal Plate Agglutination Test and STA Test are the primary methods to diagnose brucellosis, especially in patients with negative bacterial culture results. Laboratory data included CRP, ESR, leucocyte count, neutrophil count, lymphocyte count, monocyte count, eosinophilic count, basophil count, erythrocyte count, hemoglobin count, hematocrit, platelet count, and thrombocytocrit.

\section{Imaging protocol}

All patients underwent pectoral CT scans within three days after admission. CT scans were created using a 64-speed multi-scan system (High Speed; GE Medical Systems, Milwaukee, WI, USA, and Light Speed; GE Medical Systems, Milwaukee, WI, USA). Unenhanced CT had been processed with numbers: $120 \mathrm{kVp}$, $150 \mathrm{~mA}, 5-\mathrm{mm}$ thick. Thin-section CT images have been reconstructed using 1-mm or 1.25-mm collimation, which have been evaluated using a lung window, 1600-window width, and 600-window level, and then send to the workstation (ADW 4.2; GE Medical Systems, Milwaukee, WI, USA) by analysis.

Taking into account standard morphological descriptors based on the recommendations of the study [14], the examination of thin-slice CT abnormalities included the distribution of lesions, small nodule, patchy shadow, consolidation, interlobular septal thickening, lymph node enlargement ( $\geq 5 \mathrm{~mm}$ ), pleural thickening, pleural effusion, pericardial thickening, pericardial effusion. Two senior radiologists who worked at least 18 years arranged to analyze their chest images severally to find out and record all the lesions. When two radiologists were not in agreement with each other, the consensus was reached through consultation.

\section{Statistical Methods}

All of the data were put into our computer database. The median was applied to analyze the continuous variables, which presented abnormal distribution statistically, and the percentage was used to analyze the classified variables, respectively.

\section{Results}

\section{Demographic characteristics}

The demographic characteristics of PB patients were shown in table 1. There were 1376 patients with brucellosis from January 2011 to January 2020, among which 38 (2.8\%) patients were diagnosed with PB. Of the total 38 patients, 26 were males and 12 were females (about 2:1 ratio). The mean age was $41.4 \pm 13.3$ years (range 17- 75 years) and the median age was 51 years. It seemed that the most vulnerable age range was 40-59 years. 18 (47.4\%) patients were Uygur and $10(26.3 \%)$ were Han. 29 (76.3\%) were herdsmen who worked with livestock all day and 11 (28.9\%) patients suffered from diabetes. 
Table 1

The demographic characteristics and clinical manifestations of the patients with PB $\mathrm{PB}(\mathrm{N}=38)$

Number\n囚

Percentage $\% \bigotimes$

Sex

Male

26

68.4

Female

12

31.6

Age

0-19

2

5.3

20-39

5

13.2

40-59

24

63.2

60-79

7

18.4

Nationality

Uygur

18

47.4

Han

10

26.3

Kazak

4

10.5

Mongolian

4

10.5

Others

2

5.3

Occupation

Herdsmen

29

76.3

Workmen

4

10.5

Others

5

13.2

Clinlcal symptom

Fever

28

73.7

Fatigued

26

64.4

Loss of appetite

22

57.9

Loss of weight

21

55.3

Lumbago

16

42.1

Sweating

12

31.6

Joint pain

11

28.9

Page 5/16 


\begin{tabular}{|c|c|c|}
\hline Cough and sputum & 9 & 23.7 \\
\hline Headache & 7 & 18.4 \\
\hline Diarrhoea & 6 & 15.8 \\
\hline Muscular soreness & 5 & 13.2 \\
\hline Nausea and vomitting & 5 & 13.2 \\
\hline Pharynx & 4 & 10.5 \\
\hline Chest tightness & 3 & 7.9 \\
\hline Erythema & 3 & 7.9 \\
\hline Chest pain & 2 & 5.3 \\
\hline Dry eye & 2 & 5.3 \\
\hline Ventosity & 2 & 5.3 \\
\hline Lower limb edema & 2 & 5.3 \\
\hline Itch of skin & 2 & 5.3 \\
\hline Frequent urination & 1 & 2.6 \\
\hline Jaundice & 1 & 2.6 \\
\hline Hematuresis & 1 & 2.6 \\
\hline Scrotal swelling & 1 & 2.6 \\
\hline Testiculus pain & 1 & 2.6 \\
\hline \multicolumn{3}{|l|}{ Diabetes } \\
\hline Positive & 11 & 28.9 \\
\hline Negative & 24 & 63.2 \\
\hline Not mentioned & 3 & 7.9 \\
\hline
\end{tabular}

\section{Clinical characteristics}

The clinical manifestations of PB were shown in table 1. Amongst the 38 patients, the most common clinical symptom was fever (73.1\%), containing 20 patients whose maximum body temperature exceeded $38.5^{\circ} \mathrm{C}$. More than half of the patients endured fatigued (64.4\%), loss of appetite (57.9\%) and loss of weight (51.3\%). And the lost weight ranged from $2 \mathrm{~kg}$ to $19 \mathrm{~kg}$ within six months since they got sick. 
Incredibly, only a minor part of patients showed chest symptoms like cough and sputum (23.7\%), chest tightness (7.9\%), chest pain (5.3\%). The symptoms like lumbago or joint pain might suggest complications in other parts of the body.

\section{Laboratory results}

The laboratory data of PB were displayed in detail in Table 2 and figure 1. In our study, there were 19 successful germiculture cases after five days of incubation, of which Br.melitensis infected 100\%. 31 patients showed positive results in Bengal Plate Agglutination Test and 32 showed positive results in STA Test.

Table 2

The laboratory data of patients with PB

\begin{tabular}{|c|c|c|}
\hline & Number (n) & Percentage (\%) \\
\hline \multicolumn{3}{|l|}{ Bacterial Culture } \\
\hline Brucella melitensis & 19 & 50.0 \\
\hline Negative & 19 & 50.0 \\
\hline \multicolumn{3}{|c|}{ Bengal Plate Agglutination Test } \\
\hline Positive & 31 & 81.6 \\
\hline Negative & 7 & 18.4 \\
\hline \multicolumn{3}{|l|}{ STA Test } \\
\hline Positive & 32 & 84.2 \\
\hline Negative & 6 & 15.8 \\
\hline Anaemia & 30 & 78.9 \\
\hline Male & 20 & 52.6 \\
\hline Female & 10 & 26.3 \\
\hline
\end{tabular}

Almost all patients had CRP (94.7\%) and ESR (97.4\%) raised. More than half of the patients had a low eosinophilic count (52.6\%), a low erythrocyte count (65.8\%), a low hemoglobin count $(78.9 \%)$ and a low hematocrit (84.2\%). The anemia haunted almost all female patients (83.3\%) and most male patients (76.9\%).

\section{Imaging findings}


The imaging findings of PB were displayed in Table 3. On chest CT, all patients had abnormalities. 27 patients had pulmonary nodules, including 19 (70.4\%) patients in both lung fields (Fig. 2), 7 (29.9\%) patients in the right lung, and 1 (3.7\%) patients in the left lung. These nodules' size was not significant, ranging from $3 \mathrm{~mm}$ to $17 \mathrm{~mm}$. $23(60.5 \%)$ cases were interstitial infiltration, showing in both lung. 16 patients had consolidation, including 12 (70.4\%) both lungs, 2 (29.9\%) the right lung (Fig. 3), and 2 $(29.9 \%)$ the left lung. 15 cases revealed patchy shadow, including $7(46.7 \%)$ patients in the right lung, 5 (33.3\%) patients in the left lung (Fig. 4), and 3 (20.0\%) patients in both lung fields. Of the 7 patients with atelectasis, 4 were located in both lungs, 2 in the right lung, and 1 in the left lung.

Table 3

The Imaging findings of lung with PB patients

\begin{tabular}{|c|c|c|c|c|c|c|c|c|c|c|}
\hline & \multicolumn{2}{|c|}{ Interstitial infiltration } & \multicolumn{2}{|c|}{ Patchy shadow } & \multicolumn{2}{|c|}{ Consolidation } & \multicolumn{2}{|c|}{ Nodule } & \multicolumn{2}{|c|}{ Atelectasis } \\
\hline & $\mathrm{n}$ & $\mathrm{P}(\%)$ & $\mathbf{n}$ & $\mathrm{P}(\%)$ & $\mathbf{n}$ & $\mathrm{P}(\%)$ & $\mathbf{n}$ & $P(\%)$ & $\mathbf{n}$ & $\mathrm{P}(\%)$ \\
\hline Left lung & 0 & 0 & 7 & 18.4 & 2 & 5.3 & 1 & 2.6 & 1 & 2.6 \\
\hline Right lung & 0 & 0 & 5 & 13.2 & 2 & 5.3 & 7 & 18.4 & 2 & 5.3 \\
\hline Both lungs & 23 & 60.5 & 3 & 7.9 & 12 & 31.6 & 19 & 50.0 & 4 & 10.6 \\
\hline
\end{tabular}

The findings outside the lung with PB patients were shown in Table 4. 11 patients had pleural thickening and $10(26.3 \%)$ patients suffered from hydrothorax. 20 (52.6\%) patients had lymphadenectasis in the hilum of lung or mediastinum, three pericardial effusion, and 1 pericardial thickening. The lesions accompanied by other sites were the lumbar spine (18.4\%), sacroiliac spine (10.5\%), liver (7.9\%), spleen (7.9\%), brain (5.2\%), thoracic spine (2.6\%), ankle (2.6\%), and epididymis $(2.6 \%)$. 
Table 4

The findings outside the lung with PB patients

\begin{tabular}{|lll|}
\hline & Number(n) & Percentage(\%) \\
\hline Pleural thickening & 11 & 28.9 \\
\hline Left side & 2 & 5.3 \\
\hline Right side & 4 & 10.5 \\
\hline Both sides & 5 & 13.2 \\
\hline Pleural effusion & 10 & 26.3 \\
\hline Left side & 2 & 5.3 \\
\hline Right side & 1 & 2.6 \\
\hline Both sides & 7 & 18.4 \\
\hline Pericardial thickening & 1 & 2.6 \\
\hline Pericardial effusion & 3 & 7.9 \\
\hline Lymphadenectasis & 20 & 52.6 \\
\hline Accompanied by other sites & & \\
\hline Lumbar vertebrae & 7 & 18.4 \\
\hline Lumbosacral vertebrae & 4 & 10.5 \\
\hline Liver & 3 & 7.9 \\
\hline Spleen & 3 & 7.9 \\
\hline Brain & 2 & 5.2 \\
\hline Thoracic vertebrae & 1 & 2.6 \\
\hline Ankle & 1 & 2.6 \\
\hline Epididymis & 1 & \\
\hline Note: PB=Pulmonary brucellosis & & \\
\hline & & \\
\hline
\end{tabular}

\section{Treatment}

Patients were treated on regimens of various combinations of antibiotics. 21 patients started with rifampicin and doxycycline; five patients started with isoniazid, rifampicin, and doxycycline, five patients with other antibiotics like compound sulfamethoxazole last for 6 weeks to 8 weeks. After treatment with anti-brucella drugs, clinical symptoms disappeared in all patients, and thoracic manifestations disappeared in 33 patients, improved in 5 patients. 


\section{Discussion}

As far as we can know, this is the biggest series of brucella encroached on lungs reported in China and it is considered that it was the adult Uighur men who seemed more susceptible to PB, especially 40 to 59 years old. The herdsman was the most dangerous profession as to PB. Fever, especially the irregular high fever, were the most common symptoms and the respiratory system's manifestations were less instead. The scattered pulmonary nodules and pulmonary consolidations were the most conspicuous CT findings.

In the previous literature, the incidence of PB has been inconsistent. In the current study, PB's rough morbidity rate was $2.76 \%$ matching the conclusions drawn by Wallach JC et al. [8]. However, a survey described $8.2 \%$ of patients who were involved in PB [15], in which the rough morbidity rate of PB was almost three times as ours. The reason may be that our previous understanding of PB was insufficient, and in consideration of misdiagnosis, the actual PB rates may be higher than reported.

PB is a peculiar pulmonary infectious disease caused by brucella and its unambiguous mechanism remains unclear. In this study, especially 40 to 59 years old, most of them were adult male herders, about twice as many women, which showed a fantastic resemblance to Pappas $\mathrm{G}$ et al. [16]. And Sevilla López $S$ et al. [17] agreed with our conclusion too. The contaminated aerosols may be responsible for it [3]. The unbalanced population distribution could expound the findings above. As we all know, male workers are in the majority at the places where stockbreeding thrives, especially in their prime (30-50 years). Moreover, brucella usually infects animals, hidden in their viscera and blood and the infected livestock show few clinical manifestations, except the pregnant animals. The pregnant livestock tends to miscarry or deliver a stillbirth, leaving a large number of brucella in the amniotic fluid, placenta and secretion contain. The herders work without gloves, masks, goggles, and their respiratory mucosa, their eyes and even their fresh wound are exposed to contaminated aerosol and unsterilized implements directly $[9,18]$. That might be the main crime culprit to the high incidence among the herders. The habit of ingesting raw milk should also blame [19]. Besides, in the current, $28.9 \%$ of patients suffered from diabetes, which could indirectly weaken the immune system and lead to pulmonary infection.

To our knowledge, there were a few documents that mentioned PB by far and the symptoms like fever, fatigue, loss of appetite, pectoralgia, chronic cough and chest pain were usually reported [20]. The previous studies concerning brucellosis consented to our findings $[9,17,21,22]$. It is noteworthy that only a minor part of our study patients got troubled with chest symptoms, which led to missed diagnosis without radiological examination.

In this study, most patients had significant laboratory indicators, and changes and showed that the most common abnormalities were the raised CRP, consistent with the published literature $[15,23]$. What was more, the low eosinophilic count, low erythrocyte count, low hemoglobin count and low hematocrit could be observed among the majority of cases, and Zheng $\mathrm{R}$ et al. [4] got similar conclusions with us. The thrombocytopenia can be detected in some patients, and a similar finding had been reported by Trotta A et al. [24]. Though the clear machinery of self-regulation was still unknown, it may have something to do with the role platelets played in the immune response. Although the blood bacteria culturing was 
recognized as a gold standard of the diagnosis of brucellosis [25], it was hard to make it due to its fastidious nature [26]. In our study, all the patients had blood cultured. Only $50 \%$ of cases were positive of Brucella melitensis, indicating that the low success rate should be responsible for the inappropriate diagnosis or delayed diagnosis. The Bengal Plate Agglutination Test and STA Test were emphasized to diagnose brucellosis, especially in those patients with negative bacterial culture results. The view was vividly demonstrated in this study.

Due to the high contrast, the pectoral CT examination is essential to find and surveillance the lungs' lesions. The pulmonary nodules are frequently seen on physical examination in the patients with PB, especially in the right lower lobe, and the calcification was observed in some pulmonary nodules. In our series, pulmonary interstitial infiltration and multiple intrapulmonary nodules were found in more than half of the patients. The pulmonary consolidations in the lungs were also seen in some patients, which seemed similar to pneumonia and tuberculosis. Though brucellosis can lead to bilateral hilar lymphadenopathy and mediastinal lymphadenopathy, it hardly causes necrosis in the enlarged lymph nodes. This may be the key point to differentiate PB from lymphatic metastasis. When brucella attacks the pleura, it causes pleural effusion, in most cases are bilateral. Pourbagher MA et al. [12] approved of our viewpoints. Our study also revealed the lesions accompanied by other sites were the most common lumbar spine, following by lumbosacral spine, liver, spleen, thoracic spine, ankle, and epididymis.

There exist some limitations in our research. First of all, the number of patients is limited and the sample size needs to be expanded. Then, as the patients were picked from the same general hospital, selective bias appeared inevitably. What was more, the clinical follow-up was essential and it should be done.

In brief, the incidence of respiratory system involvement is pretty low in brucellosis. PB may exist as an isolated asymptomatic pulmonary disease or may appear with complications from head to foot.

Nevertheless, even though the chest symptoms are absent, PB should be considered a possible diagnosis when CT shows pulmonary nodules and pneumonia, especially in endemic areas.

\section{Abbreviations}

PB Pulmonary brucellosis

CT Computed tomography

STA Standard Tube Agglutination

ESR Erythrocyte sedimentation rate

CRP C-reactive protein

\section{Declarations}


This study was performed by the principles of the declaration of Helsinki. Ethical approval for the study was obtained from the ethical review committee for the first affiliated hospital of Xinjiang medical university(approval number: 20170214-111). All participants provided written informed consent.

\section{Consent for publication}

All authors and All participants declare consent to publication.

\section{Availability of data and material}

Our data and images support the usability of our articles.

\section{Competing interests}

None

\section{Funding}

The financial support was provided by Xinjiang Uygur Autonomous Region Natural Science Foundation of China (Grant no. 2017D01C300).

\section{Authors' contributions}

H.G. and S.L. conceived the idea. H.G. and S.L. wrote the main manuscript text, and S.L. prepared figures 1-4. S.L. and D.C. and Y.H. collected the data. H.G. and S.L. and W.L. and Y.H. performed the literature search. All authors reviewed the manuscript. All authors approved the final version for submission.

\section{Acknowledgements}

The authors gratefully acknowledge Xinjiang Uygur Autonomous Region Natural Science Foundation of China (Grant no. 2017D01C300).

\section{References}

1. Bodenham RF, Lukambagire AS, Ashford RT, Buzz JJ, Cash-Goldwasser S, Crump JA, et al. Prevalence and speciation of brucellosis in febrile patients from a pastoralist community of Tanzania. Sci Rep 2020; 10(1): 7081.

2. Kawakami N, Wakai Y, Saito K, Imaoka K. Chronic Brucellosis in Japan. Intern Med 2019; 58 (21): 3179-3183.

3. Solera J, Solís García Del Pozo J. Treatment of pulmonary brucellosis: a systematic review. Expert Rev Anti Infect Ther 2017; 15(1): 33-42.

4. Bialer MG, Sycz G, Muñoz González F, Ferrero MC, Baldi PC, Zorreguieta A. Adhesins of Brucella: Their Roles in the Interaction with the Host. Pathogens 2020; 9(11): 942. 
5. Zheng R, Xie S, Lu X, Sun L, Zhou Y, Zhang Y, et al. A Systematic Review and Meta-Analysis of Epidemiology and Clinical Manifestations of Human Brucellosis in China. Biomed Res Int 2018; 2018: 5712920.

6. Ren J, Peng Q. A brief review of diagnosis of small ruminants brucellosis. Curr Med Chem 2020 Dec 13.Online ahead of print.

7. Pappas G, Papadimitriou P, Akritidis N, Christou L, sianos EV. The new global map of human brucellosis. Lancet Infect Dis 2006; 6(2): 91-99.

8. Wallach JC, García JL, Cardinali PS, Seijo AP, Benchetrit AG, Echazarreta SE, et al. High Incidence of Respiratory Involvement in a Cluster of Brucella suis-Infected Workers from a Pork Processing Plant in Argentina. Zoonoses Public Health 2017; 64(7): 550-553.

9. Buzgan T, Karahocagil MK, Irmak H, Baran Al, Karsen H, Evirgen O, et al. Clinical manifestations and complications in 1028 cases of brucellosis: a retrospective evaluation and review of the literature. Int $\mathrm{J}$ Infect Dis 2010; 14(6): e469-e478.

10. López-Santiago R, Sánchez-Argáez AB, De Alba-Núñez LG, Baltierra-Uribe SL, Moreno-Lafont MC. Immune Response to Mucosal Brucella Infection. Front Immunol 2019; 10: 1759.

11. Tuon FF, Gondolfo RB, Cerchiari N. Human-to-human transmission of Brucella - a systematic review. Trop Med Int Health 2017; 22(5): 539-546.

12. Pourbagher MA, Pourbagher $A$, Savas $L$, Turunc T, Demiroglu $Y Z$, Erol I, et al. Clinical pattern and abdominal sonographic findings in 251 cases of brucellosis in southern Turkey. AJR Am J Roentgenol 2006; 187(2): W191-W194.

13. Esmaeilnejad-Ganji SM, Esmaeilnejad-Ganji SMR. Osteoarticular manifestations of human brucellosis: A review. World J Orthop 2019; 10(2): 54-62.

14. Wu XH, Dawei Dong DW, Ma DQ. Thin-section computed tomography manifestations during convalescence and long term follow-up of patients with severe acute respiratory syndrome (SARS). Med Sci Monit, 2016; 22: 2793-2799.

15. Barutta L, Ferrigno D, Melchio R, Borretta V, Bracco C, Brignone C, et al. Hepatic brucelloma. Lancet Infect Dis 2013; 13: 987-993.

16. Pappas G, Bosilkovski M, Akritidis N, Mastora M, Krteva L, Tsianos E. Brucellosis and the respiratory system. Clin Infect Dis 2003; 37(7): e95-e99.

17. Sevilla López S, Quero Valenzuela F, Piedra Fernández I. Bilateral pulmonary nodules due to brucellosis. Arch Bronconeumol 2011; 47(6): 320-321.

18. Almasri M, Ahmed QA, Turkestani A, Turkestani A, Memish ZA. Hajj abattoirs in Makkah: risk of zoonotic infections among occupational workers. Vet Med Sci 2019; 5(3): 428-434.

19. Dadar M, Shahali Y, Whatmore AM. Human brucellosis caused by raw dairy products: A review on the occurrence, major risk factors and prevention. Int J Food Microbiol 2019; 292: 39-47.

20. Erdem H, Inan A, Elaldi N, Tekin R, Gulsun S, Ataman-Hatipoglu C, et al. Respiratory system involvement in brucellosis: the results of the Kardelen study. Chest 2014; 145(1): 87-94. 
21. Jiang W, Chen J, Li Q, Jiang L, Huang Y, Lan Y, et al. Epidemiological characteristics, clinical manifestations and laboratory findings in 850 patients with brucellosis in Heilongjiang Province, China. BMC Infect Dis 2019; 19(1): 439.

22. Li D, Li L, Zhai J, Wang L, Zhang B. Epidemiological features of human brucellosis in Tongliao City, Inner Mongolia province, China: a cross-sectional study over an 11-year period (2007-2017). BMJ Open 2020; 10(1): e031206.

23. Jia B, Zhang F, Lu Y, Zhang W, Li J, Zhang Y, et al. The clinical features of 590 patients with brucellosis in Xinjiang, China with the emphasis on the treatment of complications. PLoS Negl Trop Dis 2017; 11(5): e0005577.

24. Trotta A, Milillo MA, Serafino A, Castillo LA, Birnberg Weiss F, Delpino MV, et al. Brucella abortusinfected platelets modulate the activation of neutrophils. Immunol Cell Biol 2020; 98(9): 743-756.

25. Barua A, Kumar A, Thavaselvam D, Mangalgi S, Prakash A, Tiwari S, et al. Isolation \& characterization of Brucella melitensis isolated from patients suspected for human brucellosis in India. Indian J Med Res 2016; 143(5): 652-658.

26. Saytekin AM, Ak S. Direct diagnosis of Brucella species through multiplex PCR formed by a new method. J Microbiol Methods 2018;154:86-94.

\section{Figures}

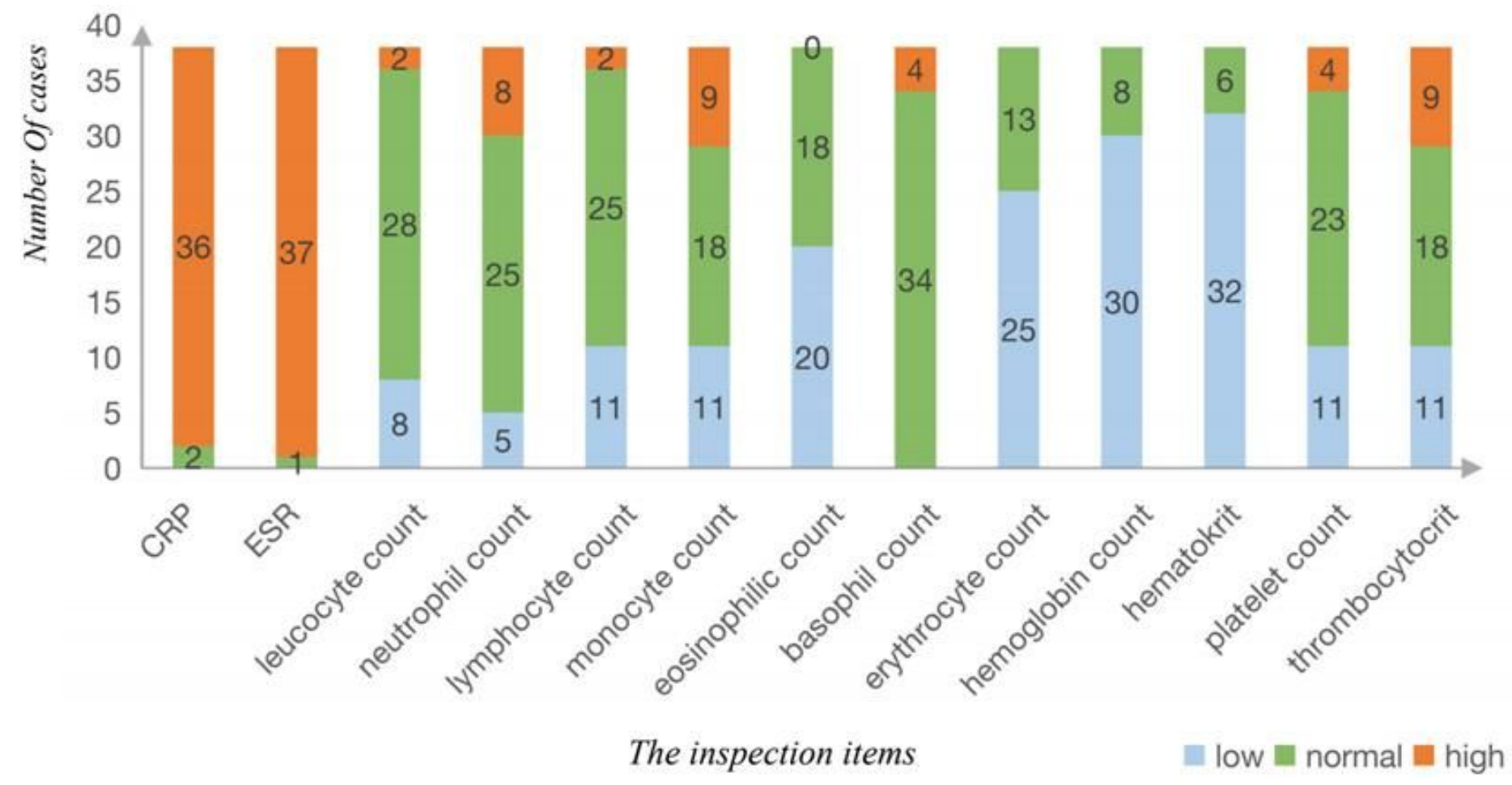

\section{Figure 1}

The laboratory findings of pulmonary brucellosis patients. 


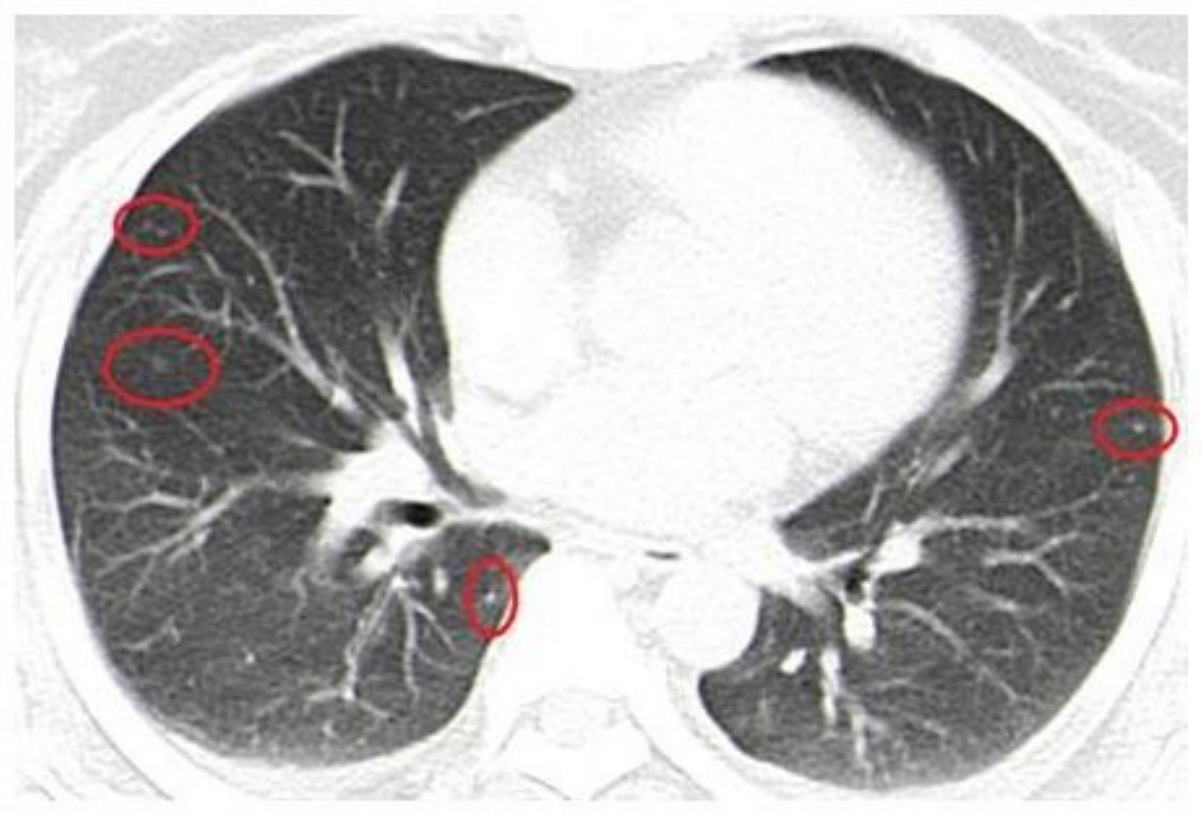

Figure 2

Axial CT demonstrated multiple nodules outside the lower lobes of both lungs.

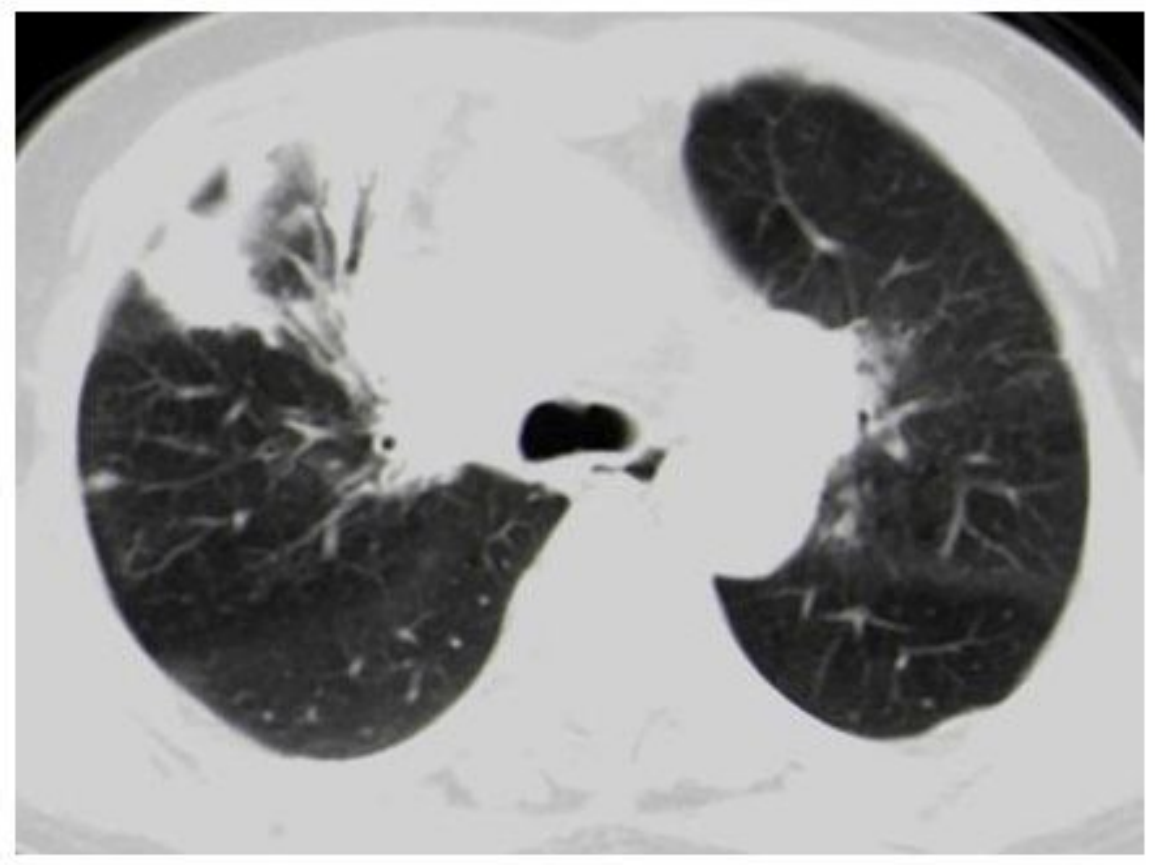

Figure 3

Axial CT revealed patchy consolidation in the middle lobe of the right lung. 


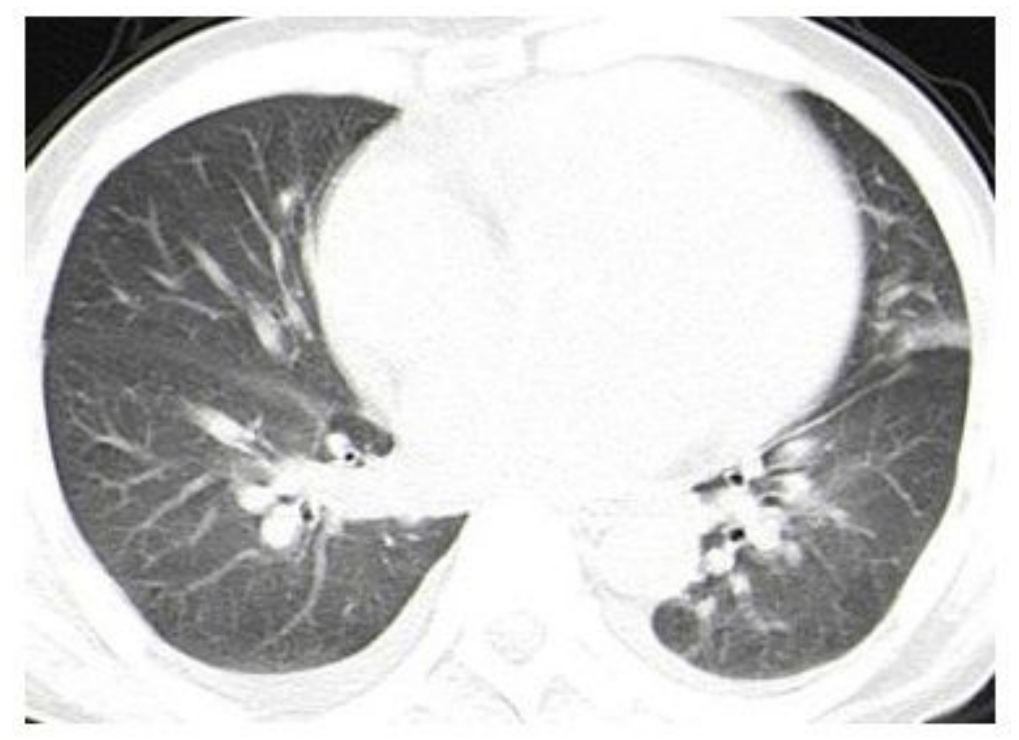

Figure 4

Axial CT showed the glossal segment of the upper lobe of the left lung is patchy. 